\title{
Inductive spikes and gamma-ray flares from the Crab Nebula
}

John G. Kirk*

Max-Planck Instute for Nuclear Physics, Saupfercheckweg 1, 69117 Heidelberg

E-mail: john.kirk@mpi-hd.mpg.de

\section{Gwenael Giacinti}

Max-Planck Instute for Nuclear Physics, Saupfercheckweg 1, 69117 Heidelberg

E-mail: gwenael.giacinti@mpi-hd.mpg.de

The $\sim 400 \mathrm{MeV}$ flaring emission from the Crab Nebula is naturally explained as the result of an abrupt reduction in the mass-loading of the pulsar wind. Very few particles are then available to carry the current required to maintain wave activity, causing them to achieve high Lorentz factors. When they penetrate the Nebula, a tightly beamed, high luminosity burst of hard gamma-rays results, with characteristics similar to the observed flares. This mechanism may operate in other powerful pulsars, such as J0537-6910 (PWN N 157B), B0540-69, B1957+20 and J0205+6449 (3 C 58).

7th Fermi Symposium 2017

15-20 October 2017

Garmisch-Partenkirchen, Germany

*Speaker. 


\section{Introduction}

One of the most interesting discoveries of the AGILE and Fermi satellites is the rapid variability of the emission at hundreds of $\mathrm{MeV}$ from the Crab Nebula [1, 2, 3]. Numerous suggested explanations have appeared in the literature, but, to date, a consensus is lacking. (For a review, see [4].) In a recent Letter we suggested that inductive, radial acceleration of particles in the pulsar wind is responsible [5]. Here we give a brief description of this mechanism, and discuss its relevance in other objects.

\section{Inductive acceleration}

Crucial to our mechanism is the idea that the pulsar wind is predominantly an electromagnetic wave, that carries along a relatively small number of charged particles, specifically, electronpositron pairs. Close to the pulsar, the density of these particles is high enough to allow the wave to be described by the equations of MHD. Consequently, we assume the wave is launched as a mildly supersonic flow that contains an oscillating, frozen-in magnetic field - a configuration commonly referred to as a striped wind. This is a widely adopted assumption (see [6] for a more detailed discussion), which has some observational support [7]. An inescapable consequence is that, at some radius, the density of charges becomes too small to justify the MHD approximation. At this point, the flow begins to accelerate $[8,9,10,11,12]$. In terms of the basic wind parameters:

$$
a_{\mathrm{L}}=\left(e^{2} L_{\mathrm{s} . \mathrm{d} .} / m^{2} c^{5}\right)^{1 / 2}=3.4 \times 10^{10} L_{38}^{1 / 2},
$$

where $L_{\text {S.d. }}=L_{38} \times 10^{38} \mathrm{erg} \mathrm{s}^{-1}$ is the spin-down power of the neutron star, and

$$
\mu=L_{\text {s.d. }} /\left(\dot{N}_{ \pm} m c^{2}\right) \text {, }
$$

where $\dot{N}_{ \pm}$is the rate at which electrons and positrons are transported into the nebula by the wind, the magnetization parameter is given by

$$
\sigma(r)=\mu / \gamma(r)-1
$$

where $\gamma(r)$ is the particle Lorentz factor, which, like $\sigma$, is a function of radius $r$. The "multiplicity" parameter $\kappa$ commonly used in modeling pair production near the pulsar [13] is roughly $\kappa=$ $a_{\mathrm{L}} /(4 \mu)$ but this should not be interpreted too literally, since a self-consistent model linking pair production with the wind parameters is not yet available.

Based on a model with cold electron and positron fluids, an approximate solution in the acceleration phase can be found [11]:

$$
u_{\perp} \approx 1 \quad \gamma \approx 2 \mu r /\left(a_{\mathrm{L}} r_{\mathrm{L}}\right) \quad \sigma \approx r_{\mathrm{L}} a_{\mathrm{L}} /(2 r),
$$

where $u_{\perp}$ is the transverse component of the four velocity of the fluids (in units of $c$ ) and $r_{\mathrm{L}}$ is the light-cylinder radius. A full, numerical integration of the radial evolution equations (see [5]) is shown in Fig. 1 the approximate solution (2.4) is valid in the range $a_{\mathrm{L}} \gamma_{\mathrm{L}} / \mu \ll r / r_{\mathrm{L}} \ll a_{\mathrm{L}}$, where $\gamma_{\mathrm{L}}$ is the initial Lorentz factor of the wind. Except exactly in the equatorial plane, the wind 


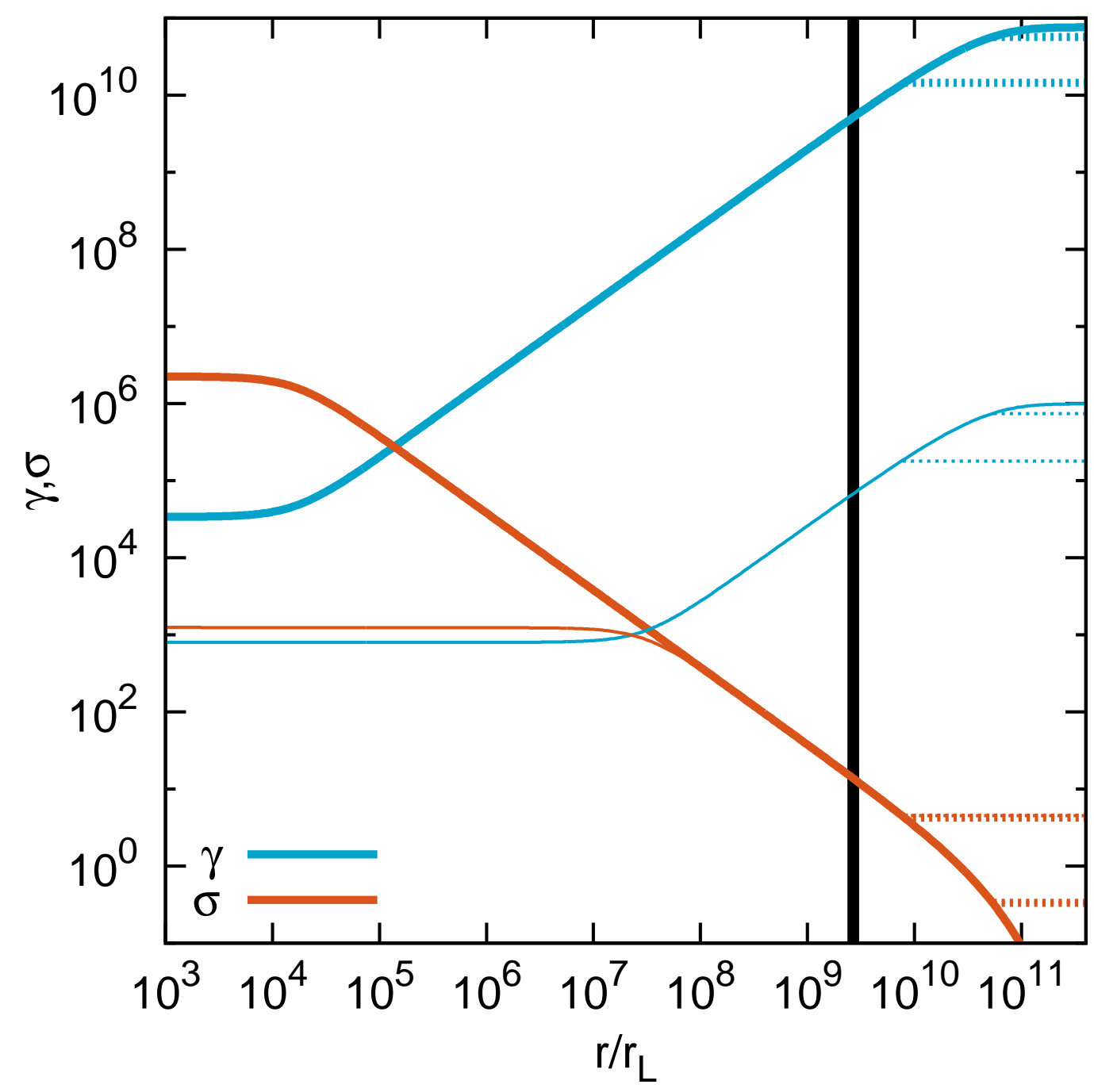

Figure 1: The magnetization parameter $\sigma$ and Lorentz factor $\gamma$ for high density $\left(\mu=10^{6}\right.$, thin lines) and low density ( $\mu=a_{\mathrm{L}}$, thick lines), with $a_{\mathrm{L}}=7.6 \times 10^{10}$ (corresponding to the $\mathrm{Crab}$ ). The thick vertical line indicates the position of the termination shock in the Crab [14].

carries with it a non-zero DC component, that cannot be converted into kinetic energy. Thus, at finite latitude, the accelerating phase of the solutions in Fig. 1 terminates when the wave energy is exhausted, even though $\sigma>0$, and the wave subsequently proceeds at constant $\gamma$ and $\sigma$, as shown by horizontal, dotted lines for initial wave amplitudes equal to $10 \%$ and $50 \%$ of the DC component at launch.

For the low-density wind, which corresponds to $\kappa \sim 1$, the electrons and positrons in the wind of the Crab pulsar achieve Lorentz factors of roughly $5 \times 10^{9}$ when they reach the termination shock, although, at this point, only about $10 \%$ of the wave energy has been converted into particle kinetic energy. 


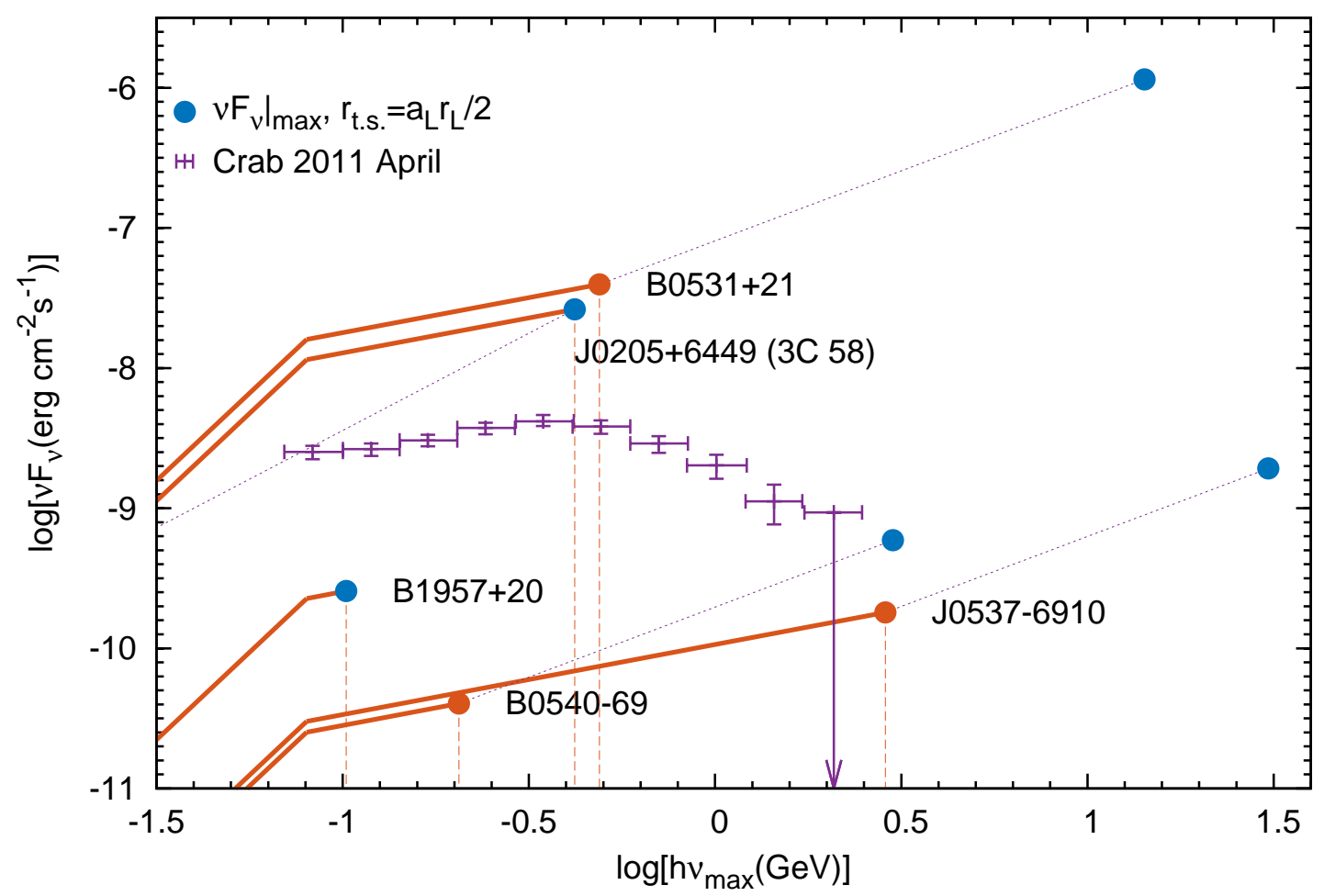

Figure 2: The predicted flare spectrum (solid lines), for the three most powerful known pulsars: the Crab $(\mathrm{B} 0531+21)$, and two objects in the Large Magellanic Cloud, assuming a turnover at $h v_{\mathrm{t}}=80 \mathrm{MeV}$ and a filling factor $f=1$. For B0531+21, J0537-6910 and B0540-69, dotted lines trace the locus of the peak flux as the position of the termination shock is varied between the observed value (orange dots) and $a_{\mathrm{L}} r_{\mathrm{L}} / 2$ (blue dots). For J0205+6449 (3C 58) and B1957+20 only the (optimistic) blue dots and the corresponding spectra are shown. Fermi observations of the powerful flare from the Crab Nebula in April 2011 are also shown - points taken from Fig 6, epoch 7 of Ref. [3].

\section{Gamma-ray flares}

On impacting the Nebula, a pocket of low density wind, with $\kappa \sim 1$, injects radially directed, $\mathrm{PeV}$ electrons into the turbulent downstream magnetic field, whose strength is roughly three times that in the wind, i.e., $B \approx 3 \times(2 \pi m c / e P)\left(a_{\mathrm{L}} r_{\mathrm{L}} / r\right)$, where $P$ is the pulsar period and $r_{\mathrm{t} . \mathrm{s}}$ is the radius of the termination shock $\left(33 \mathrm{~ms}\right.$ and $4.3 \times 10^{17} \mathrm{~cm}$ in this case). The resulting synchrotron emission peaks at

$$
\begin{aligned}
h v_{\max } & \approx 18 a_{\mathrm{L}}^{2}(h / P)\left[r_{\text {t.s. }} /\left(a_{\mathrm{L}} r_{\mathrm{L}}\right)\right] \\
& \approx 500 \mathrm{MeV}
\end{aligned}
$$

An electron initially radiating at $h v_{\max }$ is deflected by

$$
\delta \theta(v) \approx(80 \mathrm{MeV} / h v)\left(1-v / v_{\max }\right) \text { radians }
$$

whilst cooling down to emit at $h v$. Therefore, photons emitted between 400 and $500 \mathrm{MeV}$ by the electrons injected into the Crab Nebula remain radially collimated in a cone of opening angle about 
$2.5^{\circ}$. Assuming a cone of pulsar wind of solid angle $\Omega$ contains low-density pockets with volume filling factor $f$, the flux seen by an observer at distance $D$, whose line of sight lies inside this cone is

$$
v F_{v} \approx f L_{\text {s.d. }} /\left(8 \pi \sigma\left(r_{\text {t.s. }}\right) D^{2}\right)\left(v / v_{\max }\right)^{1 / 2}
$$

for $v_{\mathrm{t}}<v<v_{\max }$, where $v_{\mathrm{t}}$ is the turnover frequency below which the radiation is beamed into a solid angle significantly larger than $\Omega$. Figure 2 shows this flux for the Crab (PSR B0531+21) and the two powerful pulsars in the LMC: J0537-6910 (which may also show $\gamma$-ray flares [15]) and B0540-69.

The blue dots in this figure indicate the value of $v F_{v}$ at $v_{\max }$, assuming that $f=1$ and that the pulsar wind remains undisturbed when propagating in the direction of the observer out to the point at which $\sigma=1$, at radius $a_{\mathrm{L}} r_{\mathrm{L}} / 2$. This is clearly unrealistic for most pulsars; for the Crab $a_{\mathrm{L}} r_{\mathrm{L}} \approx 4 \mathrm{pc}$. Assuming, instead, that the wind, and, therefore, the inductive acceleration process, terminates roughly at the radius suggested by $\mathrm{x}$-ray observations [16], the maximum value migrates to the position of the orange points. In this case, a filling factor $f \approx 0.1$ gives an adequate fit to the spectrum of the powerful flare observed in April 2011[3]. The position of the $v_{\mathrm{t}}$ in this figure is arbitrarily set to $80 \mathrm{MeV}$, and the spectrum at lower photon energy is calculated assuming the electron beam diverges in the nebula to fill a cone of opening angle given by (3.2). In addition, Fig. 2 shows the value of $v F_{v}$ assuming $r_{\text {t.s. }}=a_{\mathrm{L}} r_{\mathrm{L}} / 2$ for PSR J0205+6449 (in $3 \mathrm{C} \mathrm{58)}$ ) and PSR B1957+20 (the Black Widow). In the former case, the termination shock projected onto the plane of the sky appears to lie much closer to the pulsar [16], but in the latter its position is not well constrained.

\section{Summary}

Inductive acceleration provides an attractive scenario that can be applied to flares in various astrophysical objects. It does not attempt to explain why the supply of charged particles to small parts of a magnetically dominated, relativistic outflow should suffer interruptions, but, instead, opens up the possibility of studying the physics of the electromagnetic cascades that are responsible for producing these charges. Such cascades are thought to be non-stationary, both in pulsars and in black hole magnetospheres [13, 17], but their spatial and temporal structure remains unclear. In addition, the theory makes firm predictions on the frequency dependence of the variation timescale, which should be $\propto 1 / v^{2}$, and on the polarization of the emission, which, in the pulsar case, should be that of synchrotron radiation in the predominantly toroidal nebular field.

\section{Acknowledgments}

This research was supported by a grant from the GIF, the German-Israeli Foundation for Scientific Research and Development.

\section{References}

[1] M. Tavani, A. Bulgarelli, V. Vittorini, A. Pellizzoni, E. Striani, P. Caraveo et al., Discovery of Powerful Gamma-Ray Flares from the Crab Nebula, Science 331 (Feb., 2011) 736, [1101.2311]. 
[2] A. A. Abdo, M. Ackermann, M. Ajello, A. Allafort, L. Baldini, J. Ballet et al., Gamma-Ray Flares from the Crab Nebula, Science 331 (Feb., 2011) 739, [1011. 3855].

[3] R. Buehler, J. D. Scargle, R. D. Blandford, L. Baldini, M. G. Baring, A. Belfiore et al., Gamma-Ray Activity in the Crab Nebula: The Exceptional Flare of 2011 April, ApJ 749 (Apr., 2012) 26, [1112.1979].

[4] R. Bühler and R. Blandford, The surprising Crab pulsar and its nebula: a review, Reports on Progress in Physics 77 (June, 2014) 066901, [1309. 7046 ].

[5] J. G. Kirk and G. Giacinti, Inductive spikes in the Crab Nebula - a theory of gamma-ray flares, Phys. Rev. Lett. 119 (Nov., 2017) 211101, [1710.04493].

[6] O. Porth, R. Buehler, B. Olmi, S. Komissarov, A. Lamberts, E. Amato et al., Modelling Jets, Tori and Flares in Pulsar Wind Nebulae, Space Science Reviews 207 (July, 2017) 137-174, [1703. 05184 ].

[7] M. A. McLaughlin, A. G. Lyne, D. R. Lorimer, A. Possenti, R. N. Manchester, F. Camilo et al., The Double Pulsar System J0737-3039: Modulation of A by B at Eclipse, ApJ 616 (Dec., 2004) L131-L134, [astro-ph/ 0408297 ].

[8] Y. Lyubarsky and J. G. Kirk, Reconnection in a Striped Pulsar Wind, ApJ 547 (Jan., 2001) 437-448, [arXiv:astro-ph/0009270].

[9] J. G. Kirk and O. Skjæraasen, Dissipation in Poynting-Flux-dominated Flows: The $\sigma$-Problem of the Crab Pulsar Wind, ApJ 591 (July, 2003) 366-379, [arXiv: astro-ph/0303194].

[10] H. C. Spruit and G. D. Drenkhahn, Magnetically powered prompt radiation and flow acceleration in GRB, in Gamma-Ray Bursts in the Afterglow Era (M. Feroci, F. Frontera, N. Masetti and L. Piro, eds.), vol. 312 of Astronomical Society of the Pacific Conference Series, p. 357, June, 2004, astro-ph/0302468.

[11] J. G. Kirk and I. Mochol, Charge-starved, Relativistic Jets and Blazar Variability, ApJ 729 (Mar., 2011) $104,[1012$. 0307].

[12] J. Zrake, Crab Flares due to Turbulent Dissipation of the Pulsar Striped Wind, ApJ 823 (May, 2016) 39, [1512.05426].

[13] B. Cerutti and A. M. Beloborodov, Electrodynamics of Pulsar Magnetospheres, Space Science Reviews 207 (July, 2017) 111-136, [1611.04331].

[14] J. J. Hester, K. Mori, D. Burrows, J. S. Gallagher, J. R. Graham, M. Halverson et al., Hubble Space Telescope and Chandra Monitoring of the Crab Synchrotron Nebula, ApJ 577 (Sept., 2002) L49-L52.

[15] S. Saito, D. Khangulyan, K. Hagiwara and Y. Uchiyama, GeV gamma-ray emission from PWN N 157B powered by PSR J0537-6910, in 6th International Symposium on High Energy Gamma-Ray Astronomy, vol. 1792 of American Institute of Physics Conference Series, p. 040013, Jan., 2017, DOI.

[16] O. Kargaltsev and G. G. Pavlov, Pulsar Wind Nebulae in the Chandra Era, in 40 Years of Pulsars: Millisecond Pulsars, Magnetars and More (C. Bassa, Z. Wang, A. Cumming and V. M. Kaspi, eds.), vol. 983 of American Institute of Physics Conference Series, pp. 171-185, Feb., 2008, 0801.2602 , DOI.

[17] A. Levinson and N. Segev, On the existence of steady gap solutions in rotating black hole magnetospheres, ArXiv e-prints (Sept., 2017), [1709.09397]. 